\title{
Détection du 7-aminoflunitrazépam dans de la matière cérébrale par UPLC-ToF/MS
}

\section{Identification of 7-aminoflunitrazepam in brain material using UPLC-ToF/MS}

\author{
Pascal Kintz ${ }^{\star}$, Guillaume Salquebre, Marion Villain, Vincent Cirimele \\ Laboratoire ChemTox, 3 rue Gruninger, 67400 Illkirch, France
}

\begin{abstract}
Mots clés : UPLC-ToF/MS, 7-aminoflunitrazépam, soumission chimique, encéphale
\end{abstract}
Key words: UPLC-ToF/MS, 7-aminoflunitrazepam, drug-facilitated crime, brain

Reçu le 15 avril 2008, accepté après modifications le 30 juin 2008

Publication en ligne le 31 janvier 2009

\section{Introduction}

Dans le cadre d'une expertise toxicologique, la recherche de molécules sédatives, hypnotiques ou tranquillisantes dans de la matière cérébrale en décomposition a été requise. L'échantillon provenait d'une tête humaine retrouvée dans un sac plastique et qui aurait séjourné au fond d'un puits pendant près d'une quinzaine d'années.

L'investigation a été menée par chromatographie liquide couplée à la spectrométrie de masse haute résolution avec acquisition de spectres complets. Un logiciel permet la détection et le dosage semi-quantitatif de composés dans des matrices complexes à l'aide d'une librairie établie à partir des composés standards après injection. L'identification est basée sur les critères de temps de rétention, de masse exacte, de rapports isotopiques et de présence d'ions de fragments caractéristiques.

Le couplage chromatographie liquide et temps de vol est une technologie émergente, qui permet en théorie le criblage large des xénobiotiques, à partir d'une librairie maison, basée sur l'enregistrement de spectres moléculaires avec une résolution de l'ordre de 10000 . Quelques rares applications sont disponibles à ce jour dans la littérature, essentiellement pour les urines [1-5].

\section{Matériel et méthode}

Un sac en plastique, retrouvé au fond d'un puits, contenant un crâne, partiellement fracturé nous a été remis par

^ Correspondance : Pascal Kintz, Tél : 0390400 540,

Fax : 0390400 541, pascal.kintz@wanadoo.fr les services de gendarmerie pour tenter une expertise toxicologique. Selon les enquêteurs, il pourrait s'agir de restes humains, d'une victime féminine d'un tueur en série, ayant disparu depuis une quinzaine d'années. À l'intérieur de la cavité crânienne, une masse blanchâtre à reflets verts était présente. Le sac plastique, la température basse au fond du puits, l'absence de lumière, une humidité importante sont autant de facteurs qui pourraient expliquer qu'il était encore possible de retrouver du tissu organique.

Un gramme de matière cérébrale a été prélevé. L'extraction liquide-liquide est celle pratiquée au laboratoire pour le screening toxicologique par HPLC-DAD - $1 \mathrm{~mL}$ de tampon phosphate saturé à $\mathrm{pH} 9,5$ est ajouté. L'ensemble est homogénéisé à l'aide d'un broyeur Ultraturax puis extrait par $5 \mathrm{~mL}$ d'un mélange dichlorométhane/isopropanol/ $n$-heptane (25/10/65, v:v). La phase organique, obtenue après agitation et centrifugation, est évaporée, puis reprise avec $60 \mu \mathrm{L}$ de méthanol.

L'analyse des échantillons est réalisée par UPLC-ToF/MS. Un aliquote de $5 \mu \mathrm{L}$ est injecté sur une colonne Acquity $\mathrm{C}_{18}$ HSS T3 $(2,1 \times 100 \mathrm{~mm}, 1,8 \mu \mathrm{m})$ avec un gradient acétonitrile/tampon formiate $\mathrm{pH} 3$, à un débit de $0.3 \mathrm{~mL} / \mathrm{min}$. La détection est effectuée par un détecteur de masse à temps de vol LCT Premier XE (Waters) en mode d'ionisation electrospray positif et l'analyseur en mode $\mathrm{W}$, avec une résolution à 10000 . La tension du capillaire de la source est à $200 \mathrm{~V}$ pour une tension de cône à $30 \mathrm{~V}$. La tension du tube est fixée à $7200 \mathrm{~V}$. Deux acquisitions sont menées simultanément : l'acquisition de spectres complets avec et sans fragmentation induite dans la source. L'acquisition ponctuelle d'une masse de référence grâce à la technologie LockSpray ${ }^{\mathrm{TM}}$ permet la correction des 


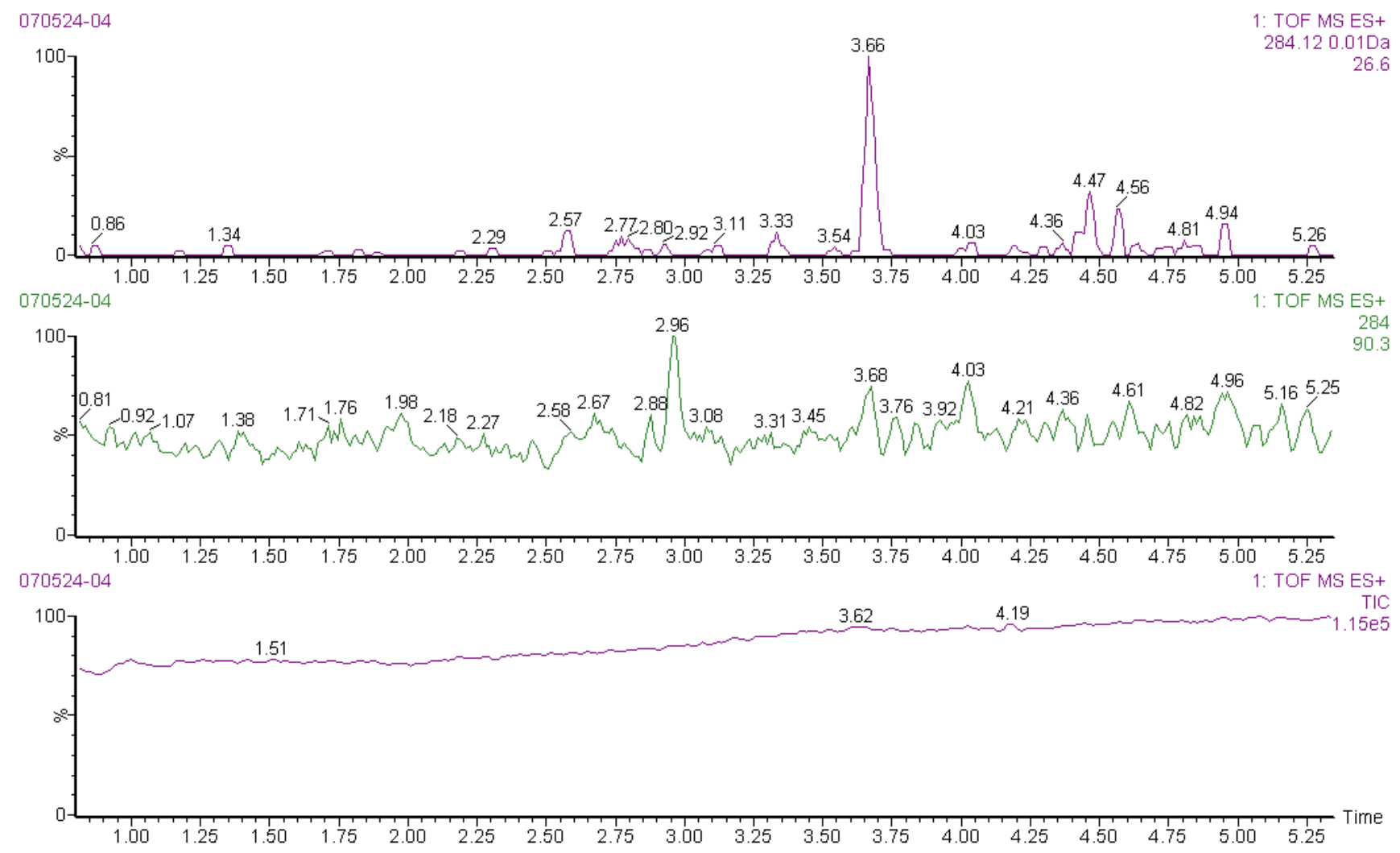

Fig. 1. Extrait d'encéphale, de bas en haut : (1) chromatogramme en courant total, (2) ion $m / z=284$ Da extrait à résolution unitaire et (3) ion $m / z=284,12 \mathrm{Da}$ à $+/-0,005 \mathrm{Da}$.

spectres de masse nécessaire lors des changements de conditions d'environnement telle que la température ambiante.

\section{Résultats et discussion}

À partir de la bibliothèque de composés sédatifs et hypnotiques établie au laboratoire après injection, la présence de 7aminoflunitrazépam a été mise en évidence grâce aux critères précédemment mentionnés et évalués automatiquement par le logiciel ChromaLynx ${ }^{\mathrm{TM}}$. Cette molécule est un métabolite du flunitrazépam, principe actif du Rohypnol, un hypnotique préconisé dans le traitement des insomnies.

La quantité observée ici est très faible. On remarque que le chromatogramme de l'ion à $m / z=284 \mathrm{Da}$, correspondant à l'ion moléculaire avec une résolution unitaire ne présente pas un signal discernable du bruit de fond. Par contre, en sollicitant la haute résolution, un pic chromatographique se détache assez nettement du bruit de fond à 3,66 minutes et permet de caractériser le 7-aminoflunitrazépam dont la masse théorique pour l'ion $[\mathrm{M}+\mathrm{H}]^{+}$est de 284,1199 Da (figure 1).

Une confirmation par UPLC-MS/MS a permis de quantifier le 7-aminoflunitrazépam à la concentration de $0,67 \mathrm{ng} / \mathrm{g}$. Cette teneur est faible mais, compte-tenu du délai post-mortem et de la durée du séjour en milieu extérieur, il est raisonnable d'imaginer qu' au moment des faits, la concentration devait être supérieure. Néanmoins, du fait de l'absence d'étude contrôlée sur l'exposition au Rohypnol ${ }^{\circledR}$ et la concentration dans le tissu cérébral, il n'est pas possible de se prononcer sur la quantité administrée.

\section{Conclusion}

Le screening large par UPLC-ToF/MS dans des matrices biologiques complexes se révèle un outil puissant de par la sensibilité et la spécificité de la technique. Il apparaît que la haute spécificité du couplage ToF permet de caractériser la présence de xénobiotiques dans des situations extrêmes, permettant de postuler sur un avenir prometteur de cette technologie. Néanmoins, la construction de la librairie nécessite l'injection des différentes molécules, car il n'est pas possible d'utiliser simplement le calculateur de masse pour discriminer de façon satisfaisante à partir de l'informatique plusieurs candidats potentiels. En effet, le logiciel propose des formules chimiques mais celles-ci sont difficilement exploitables en direct.

\section{Références}

1. ElSohly MA, Gul W, Murphy TP, Avula B, Khan IA. LC-(TOF) MS analysis of benzodiazepines in urine from alleged victims of drug-facilitated sexual assault. J Anal Toxicol. 2007; 31: 505-514. 
2. Polettini A, Gottardo R, Pascali JP, Tagliaro F. Implementation and performance evaluation of a database of chemical formulas for the screening of pharmaco/toxicologically relevant compounds in biological samples using electrospray ionization-time-offlight mass spectrometry. Anal Chem. 2008; 80: 3050-3057.

3. Kaufmann A, Butcher P, Maden K, Widmer M. Ultra-performance liquid chromatography coupled to time of flight mass spectrometry (UPLC-TOF): a novel tool for multiresidue screening of veterinary drugs in urine. Anal Chim Acta. 2007; 586: 13-21.
4. Kolmonen M, Leinonen A, Pelander A, Ojanpera I. A general screening method for doping agents in human urine by solid phase extraction and liquid chromatography/time of flight mass spectrometry. Anal Chim Acta. 2007; 585: 94-102.

5. Ojanpera S, Pelander A, Pelzing M, Krebs I, Vuori E, Ojanpera I. Isotopic pattern and accurate mass determination in urine drug screening by liquid chromatography/time of flight mass spectrometry. Rapid Commun Mass Spectrom. 2006; 20: 1161-1167. 